\title{
SÉMIOTIQUE DE L'ACTION: TEXTUALISATION ET NOTATION
}

\section{SEMIOTICS OF ACTION: TEXTUALIZATION AND NOTATION}

\author{
MARIA GIULIA DONDERO²
}

Pour Jacques Fontanille

RÉSUMÉ: Notre texte porte sur des questions méthodologiques que nous considérons comme non encore résolues en sémiotique, et notamment sur les instruments qui pourraient se révéler comme plus ou moins pertinents en vue d'une analyse des pratiques. Nous allons dans un premier temps reparcourir le débat concernant la relation entre une épistémologie du texte et une épistémologie de la pratique, ainsi que la question de l'énonciation (énonciation énoncée, énonciation en acte, praxis énonciative) qui traverse différents niveaux de pertinence de l'analyse. Nous proposerons dans un deuxième temps le concept de textualisation comme lieu de médiation entre texte et pratique : les textualisations (photographies, vidéos, prises de notes) permettraient de

1 Nous tenons à remercier de tout cœur Jean-Pierre Bertrand pour la relecture de ce texte et les conseils prodigués ainsi que les participants au séminaire de sémiotique «Sémiotique et sciences humaines II. Principe d'immanence et entour pragmatique » (20132014), notamment Pierluigi Basso Fossali et les organisateurs du séminaire: Denis Bertrand, Jean-François Bordron, Ivan Darrault-Harris, Anne Hénault. Un très grand merci aussi à Pierre Leclercq, coordinateur du projet A.R.C. Common (ULg, 2011-2014) et aux participants au projet pour la mise en commun du corpus.

2 Université de Liège et Fonds de la Recherche Scientifique - FNRS. E-mail: mariagiulia. dondero@ulg.ac.be. 
contrôler le déploiement de la pratique qui est, de son côté, éphémère et insaisissable. Dans un troisième temps nous réfléchirons sur la question de savoir comment les différentes textualisations peuvent se transposer mutuellement à travers un « croisement diagrammatisant » produisant une autre sorte de médiation entre pratique et analyse : la notation. Cette dernière ne possède pas le même statut des textualisations produites in vivo : elle fonctionne plutôt comme une reconstruction ex-post de la totalité de la pratique mettant en scène les évènements saillants ainsi que la grammaticalisation des gestes et des échanges. Plus généralement, l'objectif de ce texte est de nous interroger sur les objets légitimes de la sémiotique : quels sont les objets qu'elle peut se donner, sans trahir un principe d'immanence qui a caractérisé la sémiotique textuelle et sans rester enfermée dans une condition qui la rend inapte à analyser les pratiques et à répondre aux questionnements de l'actualité sociale et de l'orientation de la recherche en sciences humaines?

MOTS-CLÉS: Pratique; Texte; Textualisation; Enonciation; Notation.

\begin{abstract}
Our essay focuses on some methodological issues concerning the semiotic that were not solved yet, mainly the questions related to the more or less pertinent instruments according to analysis of the semiotic practices. We will initially retrace the debate about the epistemological relationship between text and action, outlining the forms of enunciative praxis through different levels of pertinence analysis (e.g. utterance, enunciation). Secondly, we propose the concept of 'textualization' as mediation between textand action: Conditions of textualization (photographs, videos, notes) that organizes and represents practices which are in themselves ephemeral and elusive. Finally, it seeks investigation of knowing how the
\end{abstract}


different textualization processes can be crossed mutually by means a "diagrammatized crossing" which produces other kind of mediatisation between practice and analysis that we call 'notation'. This analytical form of mediation between text and practice does not have the same status of in vivo textualization, but it works like an ex-post reconstruction which highlights the main gestures and exchanges while performing their grammaticalization. A more general aim of this essay is an enquiry into the legitimate objects of textual semiotics by means this question: what are the objects that can analyze without betraying its principle of immanence, but also without being confined to it and, as a consequence, being unable to analyze practices and be able to respond to topical questions on social issues and on the research in the human sciences?

KEYWORDS: Practice; Text; Textualization; Enunciation; Notation.

\section{Introduction}

Notre texte portera sur certaines questions méthodologiques que nous considérons comme non encore résolues en sémiotique, et qui concernent l'étude de l'action et de la pratique en acte. Notre objectif est d'intervenir dans le débat qui a commencé à occuper les sémioticiens il y a quelques années au séminaire de sémiotique de Paris IV-Sorbonne consacré à la relation entre texte et pratique. Il ne s'agira pas de développer une sémiotique de l'action du point de vue théorique : la littérature sur le débat texte/pratique, qui est surtout un débat sur l'immanence, est déjà assez importante et encore à creuser. Nous ne développerons pas non plus des analyses particulières de pratiques : ce texte vise surtout à problématiser l'écart entre les propositions théoriques d'une sémioti- 
que des pratiques, notamment dues à Fontanille (2008) et les outils d'analyse - qui sont encore à fabriquer ${ }^{3}$. Nous visons une contribution d'ordre méthodologique : la réflexion théorique n'a pas été suivie par des analyses portant sur de «bons corpus » et les ouvertures théoriques sur la pratique ou les propositions sur différentes formes d'immanence finissent par s'écraser sur des corpus littéraires ou en tout cas sur des textes accomplis et/ou exemplaires. Nous allons en revanche nous concentrer sur les différentes formes de textualisation de l'action et revenir sur la question des relations entre texte et pratique et plus précisément sur la relation, qui n'a pas été étudiée jusqu'à présent, entre pratique et textualisation des pratiques. La distinction entre texte et textualisation est capitale et nous la développerons au travers de la description proto-analytique d'un corpus qui sera constitué par des pratiques professionnelles en collaboration, et notamment par des pratiques collectives de conception architecturale.

Nous allons ainsi revenir sur le débat actuel qui concerne l'énonciation, et qui a accompagné les propositions de changement de paradigme disciplinaire - si on peut l'appeler de la sorte - qui d'une sémiotique de corpus stabilisés sur des supports, totalement objectivés, s'oriente vers une sémiotique qui vise à décrire le sens en acte et les pratiques quotidiennes, tant dans leur singularité (scènes) que dans leur répétition (stratégies).

Face à cet objectif, les questions que nous nous posons sont multiples : est-il suffisant d'en étudier les textualisations

3 Voir à ce propos Fontanille (2008). Pensons aussi à la littérature sémiotique produite en Italie: le numéro 5 de la revue italienne Semiotiche, coordonné par P. Basso Fossali (2006), « Testo, pratiche, immanenza », qui recueille entre autres les articles de Denis Bertrand, Anne Beyaert-Geslin, Jean-François Bordron, Jacques Fontanille, François Rastier, mais aussi aux ouvrages de Gianfranco Marrone qui défendent la textualité accomplie comme unique grandeur possible de l'analyse sémiotique. Voir à ce propos Marrone (2010). 
produites in vivo, c'est-à-dire la prise de notes, la photographie, l'enregistrement vidéo ou est-il plutôt nécessaire de constituer une notation de l'action qui reconstruise la totalité de la pratique ex post et qui met en scène les évènements saillants ainsi que la grammaticalisation des gestes, des échanges, des interventions graphiques, etc. ? Comment ne pas manquer, au travers de ces textualisations ou notations de pratiques, l'immédiateté de l'acte, la syntagmatique qui constitue et en même temps explique les motivations, le pourquoi de chaque geste dans l'action? Mais aussi comment ne pas passer à côté du sens de l'attente au cœur des échanges et de l'opacité du sens dans la complexité de son être en acte, un sens sans bords ou avec des cadres qui se renouvellent constamment ${ }^{4}$ ?

Plus généralement, notre objectif est de nous interroger sur les objets légitimes de la sémiotique : quels sont les objets qu'elle peut se donner, sans trahir un principe d'immanence et sans rester enfermée dans une condition qui la rend assez inutile pour analyser les changements sociétaux et inapte à répondre aux questionnements de l'actualité sociale et de l'orientation de la recherche en sciences humaines ${ }^{5}$ ?

Nous voudrions tenter d'esquisser les prolégomènes d'une méthode d'analyse qui ne réduise pas tout phénomène social à une textualité, et qui ne fasse pas coïncider le principe d'immanence avec la fermeture de l'objet d'analyse, ou bien

4 Dans ce travail nous ne prendrons pas en considération les commentaires des acteurs sur leurs actions mais nous sommes bien conscients de la nécessité de cette approche complémentaire, qui peut se nourrir d'entretiens de type « compréhensif » (KAUFMAN, 1996), si on veut saisir la sémantique de l'action. Notre travail se limitera pour l'instant à une exploration de l'exploitabilité des médiatisations de la pratique telles que les textualisations in vivo et la notation ex-post.

5 Voir à ce propos l'intervention de Jacques Fontanille du 4 décembre 2013 « La sémiotique face aux nouveaux défis sociétaux des SHS » au séminaire de Paris IV-Sorbonne concernant les possibles contributions de la sémiotique dans le monde de la gouvernance, de la prise de décision, du risque et des organisations. 
qui l'identifie avec le textualisme. Le danger qui se cache dans la persévérance dans cette voie est l'homogénéisation méthodologique et épistémologique de toute expérience.

Pour limiter ces risques, Jacques Fontanille (2008) distingue six types d'expérience : figurativité pour les signes, cohérence et cohésion interprétatives pour les textes-énoncés, corporéité pour l'objet, pratique pour la scène pratique, conjoncture pour la stratégie et enfin éthos pour les formes de vie. Dans sa théorie, ces six types d'expérience sont associés à six instances formelles ou plans d'immanence, hiérarchisés selon un système d'interfaces de pertinence. Envers ceux qui pensent que le principe d'immanence est trahi dès que l'on sort de l'analyse de la textualité institutionnellement figée (un tableau, un roman, un film, etc.), Fontanille (2008, p. 14) défend le principe d'immanence comme fondement de tous les plans d'immanence, et à propos du plan de la scène pratique il affirme:

Le principe d'immanence est indissociable [...] de l'hypothèse d'une activité de schématisation et de modélisation dynamique interne aux sémiotiques-objets et est l'aire d'activité immanente de cette schématisation qui doit nous indiquer pour chaque cas les limites du domaine de pertinence et non une décision a priori et tactique qui se focaliserait sur le seul texte.

La théorisation de cette schématisation dynamique, interne aux différentes sémiotiques-objets, vise à justifier un type d'immanence qui ne s'appuie pas sur les bords et les limites de la textualité mais sur une forme d'iconisation interne à la pratique, qui l'organise : cette forme d'iconisation peut, d'une certaine manière, être décrite comme une sorte de « coagulation » des forces en jeu qui, comme le dirait Jean- 
François Bordron, «prennent ensemble $»^{6}$. Il s'agit de concevoir des formes émergentes, des schèmes qui apparaissent et qui fonctionnent comme des niveaux organisateurs des différences et des contrastes: différences et contrastes ont toujours été considérés comme les fondements sûrs sur lesquels nous pouvons nous appuyer pour étudier les textes en tant que systèmes. Si l'énonciation a déjà bousculé la conception du système en ayant introduit un point de vue, la pratique demande aussi de repenser la question de l'organisation du système et de la manière dont les différences émergent des formes. Il faudrait en fait s'interroger sur le fait que, si les différences restent le fondement des analyses sémiotiques de tradition structuraliste, il faut aussi pouvoir décrire la manière dont des formes ou des schèmes émergent des différences. Autrement dit, les différences sont à saisir non pas d'un regard épistémologique en surplomb, mais par un regard qui puisse comprendre la manière dont ces différences s'organisent à travers des rythmes - et non seulement des syntagmes - qui en constitueront les formes émergeantes.

En revenant à Fontanille, et en reprenant ses mots, chaque énonciation en acte développe elle-même une activité de schématisation, voire une méta-sémiotique interne qui est en construction lors de l'observation. La question qui se pose est alors la suivante : cette activité de schématisation, est-elle encore en acte lors de l'analyse ? Faut-il que l'analyse s'accommode au modus operandi de la production de l'objet qu'on postule signifiant, c'est-à-dire à la pratique ? Doit-elle mimer l'être en acte de l'action, partager la même dynami-

6 Sur la notion d'iconicité et sur la relation entre sémiotique hjelmslevienne et sémiotique peircienne, voir Bordron (2011). Voir aussi les CR de Pierre Bordron (http://epublications.unilim.fr/revues/as/862) et de Maria Giulia Dondero dans Actes Sémiotiques (http://epublications.unilim.fr/revues/as/840) 
que de l'action à étudier en prenant en compte non seulement les actions effectuées mais aussi celles qui n'étaient que possibles en épousant une approche archéologique?

Nous essayerons de répondre à cette question dans les conclusions : pour l'instant nous pouvons affirmer que si nous suivons Fontanille sur les prolégomènes théoriques, nous nous en éloignons sur la méthodologie. Nous essayerons de voir en fait comment les différentes textualisations produites lors de l'observation de terrain démultiplient et complexifient les perspectives et les points de vue sur la pratique en acte : l'analyse devra en outre examiner la relation mutuelle entre ces textualisations, que nous pouvons considérer comme des schématisations de la pratique.

\section{Prémisses méthodologiques}

Si Fontanille (2008) avait effectivement relancé le débat sur le problème de l'immanence et sur les fondements de la discipline, son ouvrage n'a pourtant pas réussi à produire parmi les sémioticiens un regain d'intérêt pour l'étude linguistico-ethnographique des pratiques. Nous nous référons évidemment aux analyses conduites par la linguistique appliquée et à l'ethnométhodologie, par exemple promue par Charles Goodwin (1994; 1997; 2000; 2003) à l'UCLA de Los Angeles ou par Lorenza Mondada (2005) à Lyon II et ensuite à Bâle. Cette approche de l'analyse minutieuse des gestes, des mots et des échanges découle de l'analyse conversationnelle, ainsi que des travaux de Lucy Suchman (1987) et d'Edwin Hutchins (1995) sur la cognition distribuée. Toutes ces réflexions ont aussi inspiré la méthode analytique de Jacques Theureau, initiateur de l'approche « Le cours d'action $»^{7}$, proche de la 
théorie sémiotique peircienne, et longuement discutée par Fontanille (2008).

Venons-en maintenant aux approches sémiotiques de l'analyse des pratiques.

Jean-Marie Floch (1990) a peut-être été le premier parmi les élèves d'Algirdas Julien Greimas à entamer une analyse des pratiques et notamment des comportements et des attitudes des utilisateurs du métro parisien. Dans sa démarche, il a seulement en partie explicité les critères de ses choix méthodologiques lors des pratiques d'observation car il a soustrait à ses lecteurs le contrôle sur les différents types de comptes rendus des actions, à savoir les résultats des textualisations des pratiques d'observation. Nous sommes par contre convaincues qu'il faut expliciter ses propres critères d'observation pour qu'ils deviennent vérifiables : les conditions et les paramètres des enregistrements des pratiques et des témoignages des observateurs doivent être pris explicitement en compte comme outils analytiques pouvant se prêter à la répétition et à la falsifiabilité. La spécificité de chaque textualisation et de chaque notation des pratiques devrait à notre sens devenir objet explicite de description lors du processus de l'analyse elle-même, et fonctionner comme interface, voire comme intermédiaire, entre l'analyste et son objet d'étude final. Démultiplier les intermédiaires entre l'objet de sens visé et l'analyste sert non seulement à rendre contrôlable le travail de l'analyste mais aussi à rendre plus conscient l'analyste lui-même sur ses choix et à ce qui s'éloigne de toute illusion de «transparence » par rapport à la textualité. Les textualisations-médiations sont également utiles pour remettre en jeu les différentes perspectives sur la pertinence sémiotique de l'objet d'analyse. A travers la problématisation des différentes textualisations, la stratégie méthodologique de l'analyste 
- qui a été gelée depuis quelques temps sur le modèle de l'analyse textuelle - pourrait ainsi être mise en question et affinée car elle révèlerait les étapes d'un parcours allant de la production d'un texte (qui relève d'une énonciation en acte) aux simulacres de l'énonciation contenus dans le texte (qui relèvent d'une énonciation énoncée) et aux pratiques d'utilisation de ces textes au sein d'une culture donnée (domaine de la praxis énonciative).

Fontanille a sans aucun doute accompli des avancées théoriques importantes par rapport à Floch et à son analyse des utilisateurs du métro parisien car il a essayé d'intégrer les différents plans d'immanence en distinguant les différents types d'objets sémiotiques (texte, objet et pratique notamment). Il n'a pourtant pas posé la question du rôle d'intermédiaire que les textualisations et les notations des pratiques assument dans l'analyse des actions. Ce manque est d'une certaine façon justifiable par le fait que dans Pratiques sémiotiques Fontanille analyse des textes littéraires qui sont des récits de pratiques et non pas des pratiques en elles-mêmes : le problème de la textualisation ne s'est donc pas posé car les pratiques étudiées font l'objet d'histoires racontées à l'intérieur de textes accomplis. Fontanille a mis en place un modèle d'analyse de l'action mais il s'agit d'une action qui était déjà textualisée dans des textes littéraires. Il y a donc à notre sens un écart important entre les résolutions théoriques ${ }^{8}$, précieuses pour permettre à la sémiotique de devenir une discipline d'intervention, et les contraintes que Fontanille s'est auto-imposées concernant la méthodologie et le choix de corpus.

Pour poursuivre sur la voie tracée, et notamment sur

8 Nous nous référons notamment aux modalisations qui permettent à Fontanille de distinguer entre praxis, procédure, conduite, protocole, rituel, et qui sont gérées par des multiples combinaisons de la programmation et de l'ajustement. 
la relation entre les six différents types d'expérience rappelés plus haut, il faut commencer par distinguer les textes qui mettent en scène des pratiques (ce sur quoi se focalise l'attention de Fontanille) et les textualisations des pratiques (ce sur quoi nous focaliserons la nôtre).

La démarche de Fontanille consiste à partir des textes littéraires accomplis et de les « remettre en acte », d'en extraire le développement et le déploiement sans se poser la question de la traduction/textualisation/notation de l'acte. Mais lorsqu'on étudie une "vraie » pratique qui se déroule sous nos yeux à un moment donné au sein d'une culture donnée, il faut se poser le problème des types d'observation et de textualisation ou notation pertinents, et envisager la manière dont il faut visualiser le développement et les grammaticalisations locales de la pratique dans le temps : repérer ses unités, ses règles d'agencement, les syntagmes, les invariants, etc.

Les textualisations des pratiques posent en fait le problème de ce qui est nécessaire d'observer et/ou sélectionner de la pratique. Cette sélection s'opère aussi grâce aux différents médias qui traduisent la pratique et qui lui donnent une première stabilisation, par exemple l'écriture dans la prise de notes, mais aussi la photo et la vidéo. L'un des problèmes à affronter est certainement celui de la traductibilité, voire de la commensurabilité, entre les saillances et les discontinuités repérées par les différents médias utilisés dans la production des textualisations (photos, vidéos, notes). Ces textualisations pourraient d'ailleurs être conçues comme des proto-analyses : l'analyse finale devrait en fait également reparcourir le geste médiatique qui a sélectionné une certaine saisie de la pratique, sa spécificité, ses contraintes technologiques ainsi que les contraintes liées au point de vue.

Lorsque Fontanille a analysé les pratiques de la conver- 
sation à table et a étudié le développement des pratiques racontées par Aragon dans Les voyageurs de l'Impériale (1948), il les a croisées pour en extraire des types de stratégies et finalement une forme de vie; nous partirons en revanche de l'observation de certaines pratiques et notamment des pratiques de conception architecturale collaboratives, qui ont lieu en coprésence et à distance, afin d'explorer les différents degrés de densité phénoménologique témoignés par les textualisations des actions.

Outre la différence entre textes qui mettent en scène des pratiques - et qui peuvent être éventuellement utiles pour étudier une idéologie de l'action - et textualisations de pratiques, il faut pouvoir distinguer également textualisation de la pratique et notation de la pratique.

Les textualisations telles que l'enregistrement vidéo, la photographie, la prise de notes ne peuvent pas être définies comme des notations car elles restent trop « fidèles » à la pratique elle-même et à sa densité phénoménologique. Ce faisant, elles ne peuvent pas répondre aux critères de la notation qui vise la visualisation « d'en haut » de la totalité des actions, dé-phénoménalisée, et qui permet de cartographier l'émergence de chaque geste, leurs durées, leurs croisements, leurs successions. La notation ne vise pas à se présenter à l'instar d'un témoignage de la pratique mais bien au contraire comme une reconstruction de la pratique qui repère des homogénéités et des hétérogénéités parmi les configurations des gestes, les attaques (les démarches inchoatives), les moments de pause, les tempi de la durée, le type de participation corporelle des acteurs, etc. L'objectif en est l'identification de l'émergence de modules d'actions qui sont plus ou moins répétés, plus ou moins en contraste les uns avec les autres, plus 
ou moins fixes ou déformables et qui peuvent rendre visuellement évident un micro-alphabet des structures de l'action, ou au moins un ensemble de patterns.

Pour développer notre réflexion avant d'affronter le corpus choisi - les pratiques professionnelles d'une équipe d'architectes occupée dans une démarche créative en collaboration - , nous voudrions rappeler les derniers avatars de la théorie de l'énonciation qui, tant dans sa déclinaison textualiste que dans ses déclinaisons plus phénoménologiques ou liées à la description des cultures, nous apparait toujours au fondement de notre méthodologie disciplinaire.

\section{Le débat sur l'énonciation}

Les quinze dernières années, les recherches sémiotiques ont théorisé au moins trois sortes de niveaux énonciatifs selon les degrés de stabilisation (support) et de clôture (bords) de l'objet d'analyse, à savoir : l'énonciation énoncée (concernant les simulacres de la subjectivité en discours), la praxis énonciative (concernant les transformations internes à une culture et entre différentes cultures), l'énonciation en acte (relevant d'un ensemble d'actions à saisir dans leur déploiement). Ces trois déclinaisons de la théorie de l'énonciation devraient nous permettre d'examiner la manière dont chacune d'entre elles s'appuie sur une théorie générale du sens dont dépend la manière de constituer l'objet de la discipline.

Pour résumer brièvement le débat, surtout franco-italien, sur l'énonciation et les plans d'immanence, nous nous appuierons non seulement sur la théorie de Fontanille - que nous ne reparcourons pas car elle est bien connue - , mais aussi sur les propositions de Claudio Paolucci (2010) exposées dans son ouvrage paru en 2010 qui a pour titre Struttu- 
ralismo e interpretazione ${ }^{9}$.

Si la théorie de l'énonciation, généralement parlant, est la théorie de la distribution de la subjectivité à l'intérieur du discours, un des grands mérites de la sémiotique greimassienne est d'avoir essayé de décrire, via le concept d'énonciation énoncée, les simulacres de la subjectivité dans différents langages (par exemple dans le langage pictural, photographique, audiovisuel) ${ }^{10}$.

Dans son ouvrage, Paolucci prend position par rapport à cette tradition en rappelant que la subjectivité théorisée par Greimas et Courtés (1979) dans leur dictionnaire, tout en se déclarant simulacrale et énoncée, garde, à la suite de Benveniste, un je-ici-maintenant comme source et repère de tout discours. Cela reviendrait à dire que ces localisations déictiques maintiennent en vie un modèle d'analyse extralinguistique : celui de la situation de communication. De ceci découle, selon Paolucci, que la théorie sémiotique de tradition benvenistienne, qui a ses représentants en Greimas, Fontanille et Coquet, aurait péché en hiérarchisant le «je-tu » par rapport au « il » de la troisième personne. Cette « supériorité » du je-ici-maintenant, entendu comme source de toute prédication, sur la troisième personne, comprise comme résultat secondaire d'une relation je-tu in praesentia, avait déjà été dénoncée par un chercheur italien, Giovanni Manetti (1998), comme une «brèche dans le structuralisme ». Autrement dit, le je-ici-maintenant, peut-il être considéré comme une survivance de transcendantalisme et, ajouterions-nous, de logocentrisme? Et encore : la théorie de l'énonciation, entendue

9 Voir notamment le dernier chapitre, « Enunciazione ed effetti di soggettività » (PAOLUCCI, 2010, pp. 433-500).

10 Voir à ce sujet Fontanille (1989); Fossali \& Dondero (2011) et Dondero (2013). 
en tant que brèche dans le structuralisme, a-t-elle effectivement bousculé l'immanence hjelmslevienne par rapport à la logique du texte ? Ou bien l'immanence textuelle a-t-elle pu vivre à côté de cette impasse entre simulacres discursifs et situation de communication pointée du doigt par Paolucci?

La tradition sémiotique des déictiques, on le sait, se distingue du modèle de l'énonciation impersonnelle proposée par Christian Metz (1991), centré sur une subjectivité diffuse et non déictique, ainsi que du modèle de la sémiotique interprétative, peircienne, et développée en Italie par Umberto Eco (1975; 2013), Patrizia Violi (2005) et Claudio Paolucci (2010).

Il faut tout d'abord préciser que Metz, à la différence de l'école peircienne italienne, maintient une position textualiste (et spécifique du texte filmique). Quant à elle, la sémiotique interprétative ne réduit pas l'énonciation impersonnelle à une propriété de l'énoncé mais utilise la notion d'énonciation impersonnelle pour concevoir la relation entre les différents plans d'immanence présents dans le fonctionnement de l'encyclopédie - l'encyclopédie est d'ailleurs un modèle de fonctionnement du sens disposé en rhizome. La mise en fonction de l'encyclopédie, et donc de toute source d'acte qui gère l'actualisation et l'organisation des réseaux de sens, prend appui sur un événement énonciatif qui est conçu comme impersonnel.

Il y aurait deux manières de dépasser la notion d'énonciation en tant que théorie des déictiques et des embrayeurs : la première solution est celle d'une énonciation énoncée impersonnelle qui réfléchit à l'intérieur de l'énoncé un ensemble d'actes et de praxis. C'est la solution de Metz. La deuxième solution, appartenant à la sémiotique interprétative, serait de concevoir l'énonciation impersonnelle en tant que dispositif régulateur du fonctionnement de la relation entre pratiques de donation et de génération du sens à l'intérieur d'une cul- 
ture. Dans ce second cas, il ne s'agit plus d'énonciation énoncée version impersonnelle mais d'une énonciation entendue en tant que dispositif de contrôle, d'orientation et de gestion des activités d'assimilation/refus/transformation du neuf et de l'étranger dans les pratiques sociales quotidiennes : il faut la concevoir sur le modèle de la praxis énonciative qui, chez Fontanille et Zilberberg (1998), est déterminée par les modes d'existence : virtualisation, actualisation, réalisation, potentialisation.

Il ne s'agit donc pas seulement de décliner l'énonciation énoncée selon l'impersonnalité metzienne mais aussi de transposer cette impersonnalité à l'intérieur de la hiérarchie des plans d'immanence construits par Fontanille. Dans tous les cas, plus on se déplace vers le haut, dans la hiérarchie de Fontanille, du texte à la forme de vie, plus la stratification des énonciations permet une de-deictisation de l'énonciation. D’ailleurs, dans tous les plans d'immanence repérés par Fontanille (texte, objet, scène prédicative, stratégie et forme de vie), ce qui est en jeu c'est une déclinaison différente de la théorie de l'énonciation. Si dans le cas de l'énonciation énoncée nous sommes certainement encore liés à la tradition des déictiques et de la position à assigner au lecteur/observateur modèle, dans le parcours ascendant entre texte, objet, scène prédicative, c'est la relation entre une manipulation simulacrale - qui est donc une manipulation possible - et une manipulation agie qui est en jeu.

Pour être plus précis, relisons Fontanille qui, dans Pratiques sémiotiques, examine quatre niveaux de modulation de l'énonciation selon les modes d'existence. Pour expliquer l'intégration du texte à l'objet et successivement à la pratique, il propose de prendre en considération les tablettes d'argile de l'ancien Moyen-Orient : 
Le cas des objets est significatif du principe sur lequel repose l'ensemble du parcours envisagé : un principe d'intégration progressif par l'intermédiaire des structures énonciatives. En effet, le texte-énoncé présente deux plans d'énonciation différents : (i) : l'énonciation "énoncée », inscrite dans le texte et sur la tablette, et (ii) l'énonciation présupposée, qui reste virtuelle et hypothétique ; c'est alors l'objet-support, avec sa tablette à inscrire [...] qui va « incarner » et manifester par ses propriétés matérielles, le type d'interaction énonciative pertinent (ici : proposer/accepter, puis contester/vérifier/arbitrer). Bref, l'objet-support d'écriture intègre le texte en fournissant une structure de manifestation figurative aux divers aspects de son énonciation [...]. Par ailleurs, en tant que corps matériel, cet objet est destiné à des pratiques et les usages de ces pratiques qui sont eux-mêmes des énonciations de l'objet ; à cet égard, l'objet lui-même ne peut porter que des traces de ces usages (inscriptions, usure, patine, etc.), c'est-à-dire des « empreintes énonciatives », leur "énonciation-usage » restant pour l'essentiel, et globalement, virtuelle et présupposée : il faudra donc là aussi passer au niveau supérieur, celui de la structure sémiotique des pratiques, pour trouver des manifestations observables de ces énonciations (FONTANILLE, 2008, pp. 23-24, nous soulignons).

Dans cet extrait, Fontanille énumère plusieurs déclinaisons d'énonciation :

l'énonciation énoncée : il s'agit de l'énonciation virtualisée sous formes de marques dans l'énoncé : ce sont les simulacres de la subjectivité énonciative,

l'énonciation présupposée par le texte-énoncé qui est incarnée par le support-objet - et qui, à son tour, présélectionne le type d'interaction énonciative pertinent et possible lors des pratiques d'usage futures. Il s'agit ici d'actes d'énonciation possibles, actualisés par le support-objet qui encadre 
le texte-énoncé ;

les empreintes énonciatives déposées sur l’objet (énonciation-usage présupposé) : ce sont ces énonciations déposées sur un objet en tant que traces qui pourront s'actualiser dans des pratiques ; et enfin

les énonciation-usages manifestées effectivement dans la pratique, qui sont observables en acte, réalisées devant nos yeux lors des pratiques quotidiennes.

Ces quatre modulations du concept d'énonciation s'enchainent dans une hiérarchie : ce qui était seulement présupposé à un niveau se manifeste à un niveau supérieur comme observable et cela grâce à un processus allant de la virtualité des possibles à l'actualisation des usages. L'énonciation est donc à concevoir comme une interface modulable entre tous les niveaux de pertinence hiérarchisés.

A partir de ces propositions, il nous semble pouvoir affirmer que la voie à suivre pour rendre compte de l'action est celle de la sémiotique de l'énonciation impersonnelle, qui part de l'étude de l'acte lui-même en l'attribuant seulement ensuite à des instances énonciatives personnelles.

\section{Le Studio Digital Collaboratif et la coagulation de la pratique}

Venons-en au corpus. Il s'agit en effet de pratiques de travail en équipe et plus précisément la conception architecturale collaborative en présence et à distance.

Le groupe de recherche interfacultaire A.R.C. Common ${ }^{11}$ avec lequel nous travaillons à l'université de Liège, constitué diatisée Multimodale Naturelle (2011-2014). URL : http://139.165.65.76/common.ulg. ac.be/web/app_dev.php. 
par des ingénieurs, des architectes, des psychologues du travail et des sémioticiens, a produit et ensuite soumis à l'analyse un studio digital collaboratif ${ }^{12}$ qui permet aux architectes travaillant dans des villes lointaines de tracer ensemble, en temps réel, un même dessin en utilisant une tablette digitale partagée par les acteurs en jeu.

Le Studio Digital Collaboratif (SDC) est en fait un dispositif matériel qui associe un module externe de visio-conférence et un bureau virtuel (Figures 1 et 2), en recréant les conditions de la communication en coprésence. Chaque trait tracé par un architecte sur la table virtuelle est immédiatement visualisé sur la table située dans le siège à distance, ce qui permet à l'autre architecte d'interagir immédiatement sur le trait proposé, de le supprimer, de le modifier, etc. Tout geste graphique sur la table est enregistré par la machine elle-même.
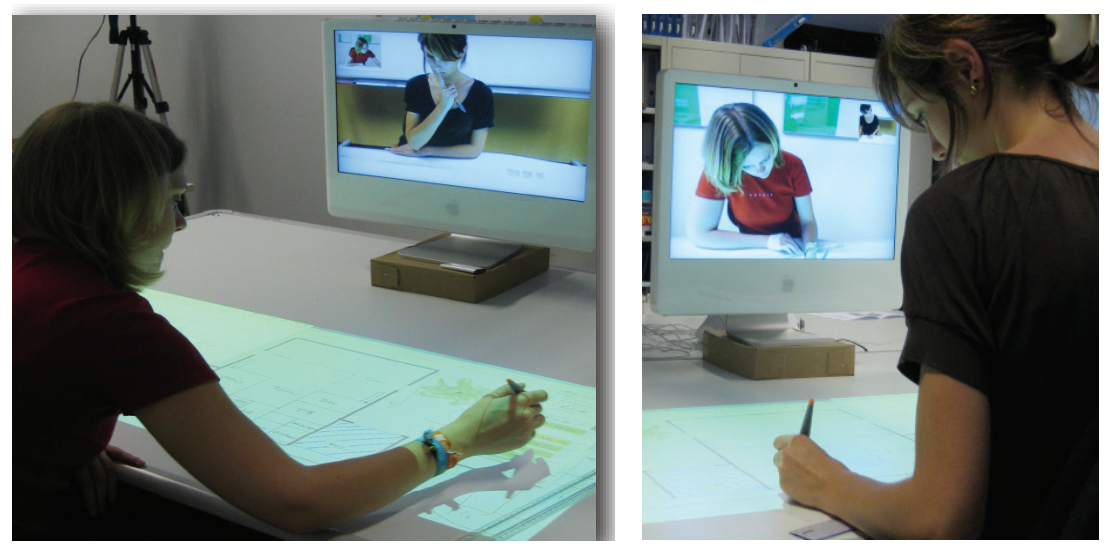

Figures 1 et 2

Dans notre démarche méthodologique, nous ne partirons pas de l'étude de la manière dont ce texte graphique

12 http://www.ulg.ac.be/cms/c_147500/fr/lucid-presente-le-studio-digital-collaboratif. Voir à ce sujet: Elsen et Leclercq (2008) ; Rajeb et Leclercq (2013). 
(dessin) est manipulé et plus généralement utilisé à l'intérieur d'une pratique ; nous partons des paramètres permettant d'étudier la pratique collective collaborative, qui se constitue dans sa globalité en tant qu'événement impersonnel.

A l'intérieur de cette pratique collaborative, nous pouvons repérer deux types de textualisation : les textualisations en acte (et plus particulièrement la production des dessins par les architectes) et les textualisations produites par l'observateur/analyste à savoir les photos, la vidéo, les notes. Ce deuxième type de textualisations, analysant et à analyser, fonctionne comme des interfaces et des médiations entre les pratiques de textualisation des dessins et l'analyste, et englobe ainsi les premières textualisations (photos, vidéos, notes). Nous les appelons textualisations car il s'agit de gestes qui se stabilisent sur un support en négociant, via un acte énonciatif, le partage entre plan d'expression et plan du contenu.

Autrement dit, cette approche méthodologique propose en premier lieu l'étude des coagulations d'actions en des textualisations, produites à l'intérieur d'une pratique professionnelle, c'est-à-dire l'étude de la transformation d'actes en traces (les dessins, les esquisses, etc.) qui se stabilisent de manière plus ou moins rapide et à travers des différents rythmes sur des supports (en l'occurrence, la table digitale). Ces textualisations produites à l'intérieur de la pratique deviennent dans un deuxième temps disponibles pour le travail de l'analyste grâce à des textualisations verbales, photographiques et audiovisuelles de niveau supérieur qui les filtrent en les mettant en perspective : elles ajoutent la perspective de la prise de vue à la perspective des dessins.

Pour les analystes, il s'agit de démêler et de débrouiller, à l'intérieur de pratiques phénoménologiquement denses de la collaboration à distance, les différents types de « coagu- 
lation » du sens qui produisent les textualisations que nous appellerons de niveau $\mathbf{n - 1}$ (les dessins et les annotations des architectes). Il faudra les étudier à travers les textualisations de la pratique (notes, photos, enregistrements vidéo) que nous appellerons de niveau $\mathbf{n + 1}$, la pratique en acte étant le niveau de départ $\mathbf{n}$. Nous sommes donc face à différents niveaux de coagulation de la pratique en des textualisations produites à l'intérieur et à l'extérieur de la pratique n. La pratique en elle-même est un événement fuyant et non-répétable : elle possède un statut autonome par rapport aux textualisations qu'elle produit (n-1) et qui sont produites à partir d'elle $(n+1)$ : elle est une dynamique d'organisation du sens ouverte, que les différents niveaux de textualisation permettent partiellement d'encadrer.

Prenons le cas d'une reprise vidéo d'une réunion de conception collaborative à distance (Figure 3).

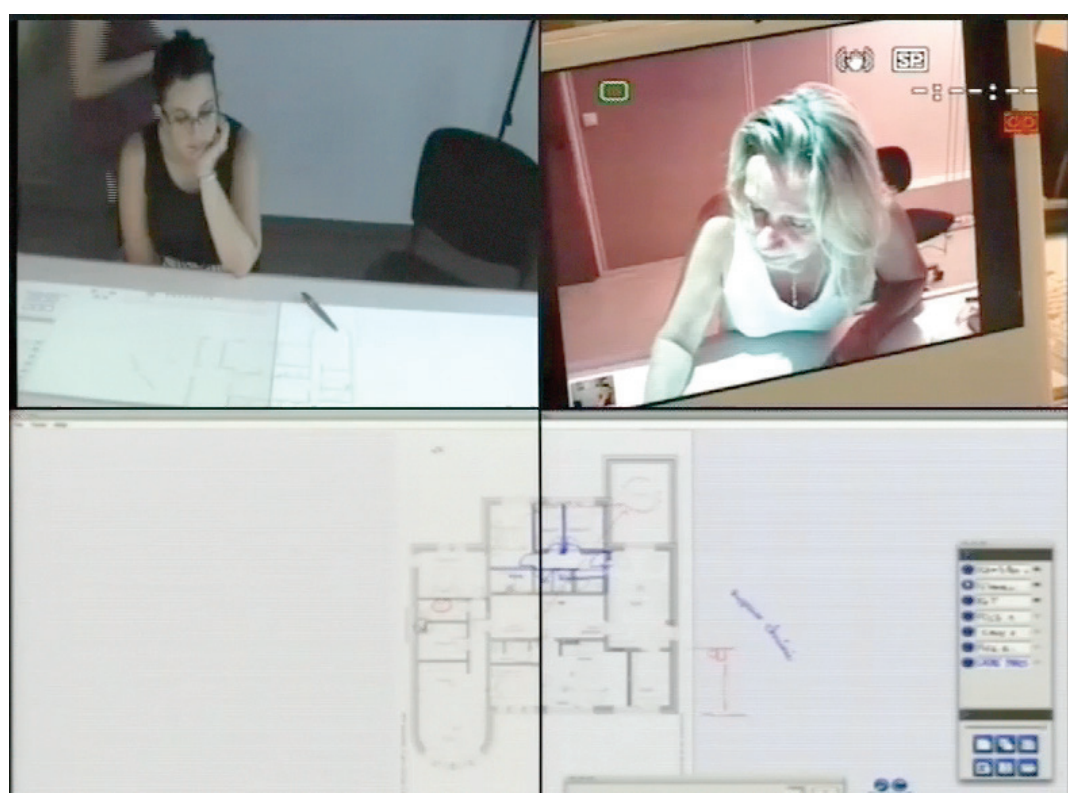

Figure 3 
Les deux cadrages en haut de la composition représentent les deux architectes occupés à la conception collaborative à distance. Ces deux images sont issues de la caméra installée sur l'ordinateur. Les deux segments visuels positionnés en bas représentent d'en haut la table digitale qui est utilisée par les deux architectes et qui est manipulable en temps réel. Les textualisations internes aux pratiques (n-1) sont identifiables avec les dessins et les annotations que le chef d'atelier et les collaborateurs produisent plus ou moins collectivement. Ces dessins sont textualisés à leur tour par les observateurs de la pratique à travers la photo, la vidéo, la prise de notes $(\mathbf{n + 1})$. Ces textualisations $\mathbf{n}+\mathbf{1}$, que l'analyste produit et qui constituent des intermédiaires entre la pratique et l'analyse, sont des prothèses de la mémoire de l'observateur, qui visualisent et organisent de manière différente la « même » pratique et qui, plutôt que la stabiliser et la réifier comme définitive et déterminée, la démultiplient à travers des perspectives médiatiques diverses.

Si les dessins et annotations, produits par les architectes, sont les textualisations-cible de la pratique de conception (n-1), les textualisations vidéo, les photos et les notes, produites par l'observateur, sont en revanche les textualisations-cible de la pratique analytique $(\mathbf{n + 1})$.

Comment pouvons-nous décrire la densité syntaxique de ces dessins (n-1) par rapport à l'acte même qui les trace ? S'agit-il de la même densité ? Non, justement : les dessins sont moins denses que les gestes car ils trouvent un ordre et une disposition sur le support qui les accueille et qui dirige et ordonne les règles et les critères d'inscription. Si les dessins « soustraient » de la densité phénoménologique aux gestes, ces textualisations, que nous qualifierons de "traçantes ", sont censées canaliser les intentions des gestes, les soumettre aux règles et aux contraintes du plan d'expression et du 
support formel ainsi qu'aux contraintes de la collaboration du collègue distant (correction/ajustement/suppression).

Les textualisations $\mathbf{n - 1}$, contrairement aux textes, sont encore manipulables et modifiables pendant la pratique en cours (et les textualisations $\mathbf{n + 1}$, d'ailleurs, les représentent in progress). Mais en même temps elles possèdent déjà un support qui les rendra suffisamment solides et stables pour pouvoir être extraites, en tant que futurs textes, de la pratique de production et être ensuite transportées vers d'autres pratiques (observation, monstration, révision, etc.). Ceci dit entre parenthèses, nous considérons les conversations entre les architectes comme des textualisations de type différent des dessins : elles s'inscrivent dans l'espace acoustique de la pratique mais elles ne sont pas ancrées dans un support qui dure : leur caractère éphémère les empêche d'être extraites de la pratique-source et d'être transportées à l'intérieur d'autres pratiques.

\section{Observations et textualisations}

Nous avons examiné le statut des textualisations n-1, à savoir les textualisations produites par la pratique de conception (les dessins et les annotations) ; venons-en maintenant plus précisément aux textualisations qui sont en revanche produites par l'observation participative $(\mathbf{n + 1})$.

Sur quelles textualisations pouvons-nous nous baser pour analyser une pratique qui est, dans ce cas, à son tour productrice de textualisations ? Comment mettre en rapport les rythmes de textualisations internes à la pratique avec les rythmes de textualisation externes à la pratique ? Autrement dit : comment mettre en relation les modalités de production des textualisations n-1 (gestes et traçage) avec les médias qui 
les textualisent $\mathbf{n}+\mathbf{1}$ (notes, photos et vidéo) ${ }^{13}$ ?

Nos observations de terrain ont précisément produit trois types de textualisation de la pratique de conception architecturale :

1. Les notes, que nous pouvons définir comme un type de textualisation caractérisé par la ponctuation et la linéarité : ponctuation au sens où les notes segmentent la pratique en valorisant des moments-clés ou bien des tendances ; linéarité car le support de l'écriture et les règles qui le soutiennent en assurent une lecture linéaire de gauche à droite.

2. La photographie, surtout dans le cas de la collaboration en coprésence, peut certainement rendre la séance avec une fidélité majeure par rapport à la prise de notes mais elle peut se révéler moins manipulable et moins flexible que l'écriture dans sa fonction proto-analytique. Comme les notes, la photo peut arrêter des moments uniques de la pratique (Figure 4) ou bien les résumer dans une vision plus globale, surtout lorsqu'une action s'arrête sur un problème ou sur une hésitation (Figure 5).

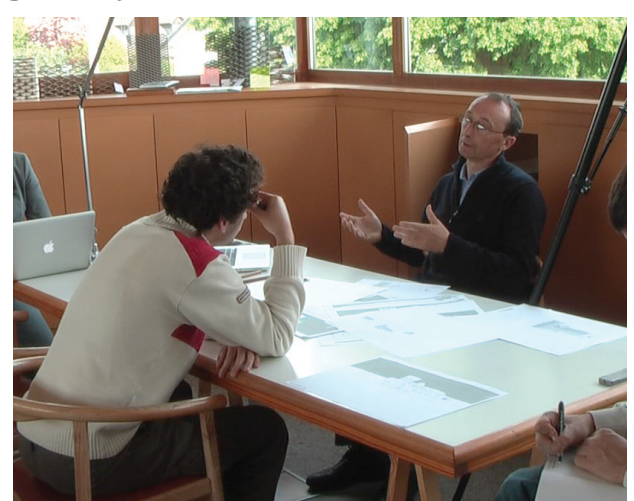

Figure 4

13 On ne peut pas considérer la mémoire de l'observateur comme une textualisation, car il s'agit d'une trace éphémère et difficile à objectiver sans les instruments des sciences cognitives. La mémoire n'a pas de densité phénoménologique stable et elle n'est donc pas valorisable dans notre travail. 


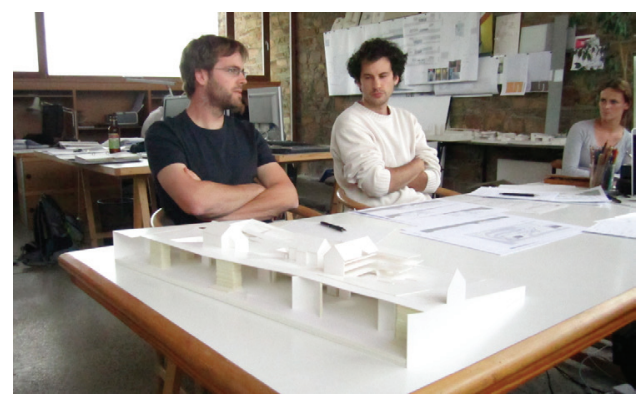

Figure 5

La photographie peut également être définie comme une textualisation ponctuelle car elle segmente, extrait, ordonne et oriente l'action de manière différente que l'action elle-même, se superpose à elle en la sélectionnant. Mais, à différence des notes, la lecture d'une photo construit un parcours tabulaire car elle se fait par sauts analogiques entre les différents centres de l'attention et les différentes saillances perceptives.

3. La vidéo, dont le point de vue est fixé en haut dans la pratique en coprésence (Figure 6), peut apparaître comme la représentation la plus fidèle car elle suit le déploiement des actes, les accompagne et les restitue au travers du filtre d'un regard surplombant, qui fait émerger tous gestes et actes des acteurs en jeu.

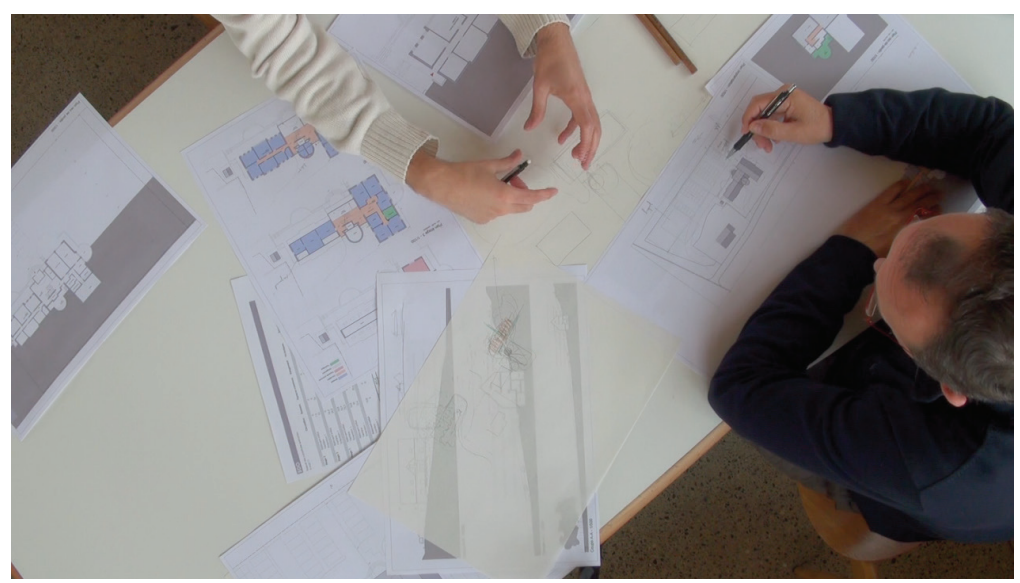

Figure 6 
L'enregistrement vidéo permet déjà une première schématisation des actes accomplis, qui sera ensuite formalisée par la notation (qui est, par contre, une visualisation/reconstruction ex-post). D'une certaine manière, nous pourrions décrire la prise vidéo comme une textualisation durative et la définir comme un lieu de rencontre entre la lecture fondée sur le parcours linéaire et celle fondée sur le parcours tabulaire. En fait, la vidéo associe la linéarité du mouvement de l'image dans le temps, qui trace un avant et un après, voire un développement, aux règles de la tabularité : la lecture linéaire est croisée par la lecture à l'intérieur de l'espace de chaque photogramme, qui procède par sauts perceptifs. La lecture de la vidéo permet un croisement entre la lecture des formes qui se transforment (ou qui restent stables) tout au long du parcours audiovisuel (linéarité et horizontalité) et les lignes de force qui émergent de la relation entre topologie du photogramme et les formes que ce dernier héberge (tabularité).

\section{La notation}

Si les trois textualisations que nous venons d'examiner sont toutes d'une certaine manière autographiques car dans tous les cas il s'agit de résultats de gestes uniques et non répétables du corps - également lorsqu'elles sont tracées par la main ou par un geste mixte, humain et technologique/digital $^{14}$, la notation par contre fonctionne de manière différente (Figure 7).

14 Sur autographie et allographie voir Nelson Goodman (1968), Languages of Art; trad. fr. Langages de l'art. Une approche de la théorie des symboles ; tr. fr. J. Morizot, Paris, Hachette, 2005. Voir aussi Dondero \& Fontanille (2012). 


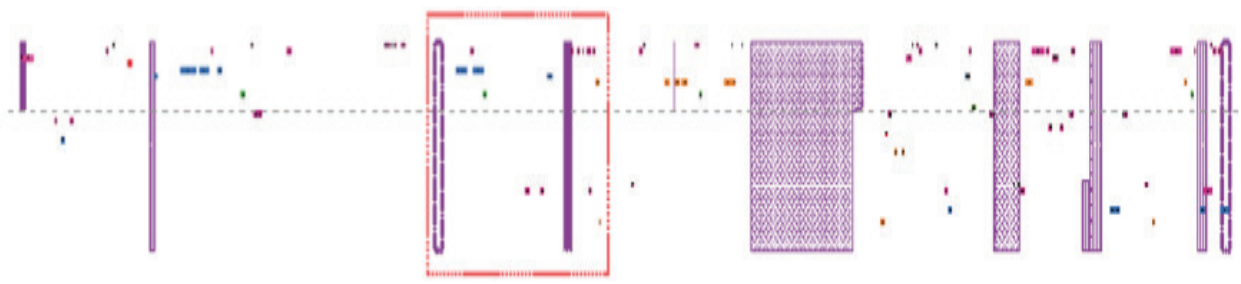

Figure 7 - Notation. Vision d'ensemble de la réunion à distance (55 min.). Encadrée en rouge une sélection de 5 min., agrandie dans l'image suivante (G. Joachim 2013).

Cette notation montre les différentes formes d'interaction graphique entre les architectes et la table digitale, leur répartition, distribution, thématisation. Cette schématisation des activités graphico-gestuelles suit deux axes : l'axe vertical marque la position des interlocuteurs (la ligne en pointillé marque la séparation physique des deux équipes et l'axe horizontal marque un référent temporel (ligne du temps). Les différentes interventions graphiques sont représentées par des segments dont la largeur indique la durée et dont la position correspond à un espace/temps précis de l'interaction (Figure 8).

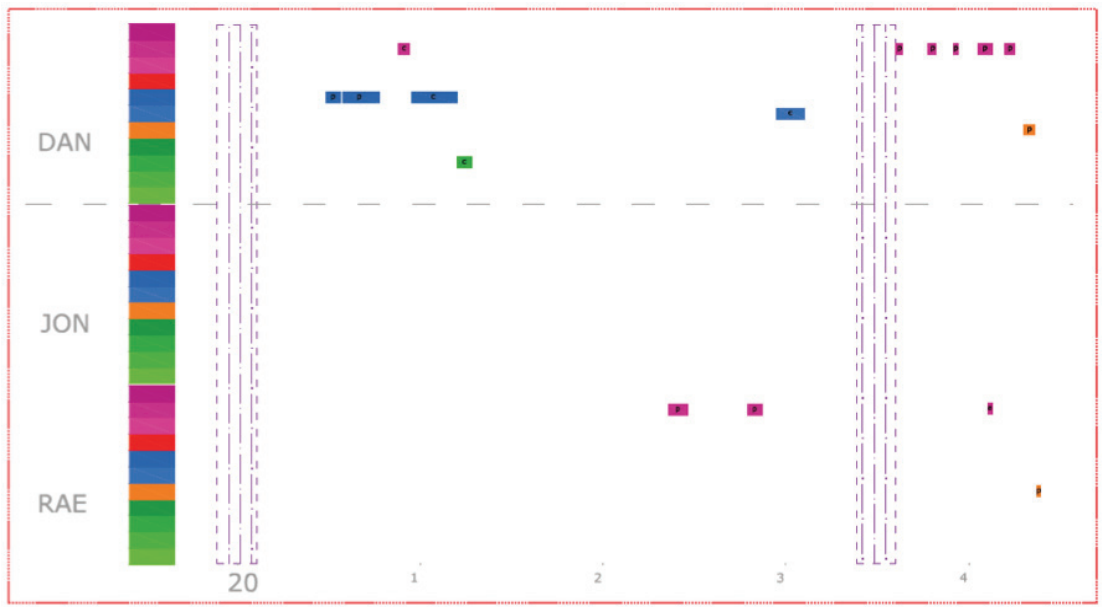

Figure 8 - Distribution des productions graphiques effectuées par DAN et RAE dans un espace de temps allant 


\section{de la minute 20 à la minute 25: dans ce temps deux mo- ments d'ajustement de l'affichage des documents sont verbalement explicités (en mauve dans la notation à la minute 20 et 23.30) (G. Joachim 2013).}

Chaque segment est coloré selon la thématisation des actes graphico-gestuels : le rouge correspond à des gestes de monstration, à des déictiques. Le bleu correspond à la figuration d'un objet, l'orange à la figuration d'un mouvement et le vert à des interventions sur des rapports d'échelle, de proportion et de point de vue. Il serait possible de noter également la différentiation, par chaque intervention graphique, du document sur lequel cette intervention est pratiquée (sur un plan, une coupe, aux marges du document, etc.). Quant aux rectangles mauves, ils sont superposés sur le schéma pour signaler les manipulations des documents graphiques, montrer les modifications de l'affichage des traits ainsi que de l'encadrement (agrandissement/réductions, etc.) ${ }^{15}$.

Cette visualisation/formalisation des interventions graphiques sur la table graphique permet de faire émerger la répartition des actions des différents architectes sur l'interface du dessin digital, ainsi que de signaler s'ils sont collectifs ou individuels.

La notation est la seule représentation de la pratique qu'on peut définir comme allographique selon les termes de Goodman, c'est-à-dire une représentation qui, contrairement aux autres textualisations, ne mime ni suit le déploiement de l'action mais sélectionne ex-post les moments de pause, de coupure, de

15 Pour une description plus détaillée de cette notation, voir Angenot et al (2013) et la thèse de doctorat en architecture de Guillaume Joachim « L'utilisation de représentations externes en conception architecturale collaborative médiée par ordinateur : le cas de la collaboration synchrone distante » dans le cadre où a été produite cette notation. 
reprise mettant enfin en scène les modules qui reconstruisent la pratique à travers la constitution des unités distinctes et la production d'un alphabet d'actions local. La notation, non seulement visualise l'ensemble des gestes, mais met en scène ses rythmes et ses cadences grâce à la constitution d'homogénéités locales qui permettent la génération de modules, lesquels sont des unités d'action : la notation offre donc une visualisation des lieux de grammaticalisation de la pratique.

\section{Conclusions}

Nous revenons, en concluant, sur la question que nous nous sommes posée au tout début : comment étudier la pratique sans en trahir la spécificité, sans la considérer comme un texte irénique, résolu, et donc en valorisant son statut d'activité et d'organisation de sens ouverte, sa disponibilité à la manipulation de la part des acteurs qu'y participent ? Et comment rendre compte de son caractère éphémère sans la rendre inaccessible, insaisissable?

Une voie possible est construire une plateforme de traductions entre les différents types de textualisation (notes, enregistrements-vidéo, photos) et la notation : nous appellerons cette plateforme une sorte de «traduction/transposition diagrammatisante ».

Nous reprenons l'acception de diagramme de Nelson Goodman (1968) qui identifie le diagramme à l'espace de transposition mutuelle et locale entre textualités fondées sur des densités syntaxiques différentes, sur différents types de valorisation du support et du point de vue, dont les polarités sont l'autographie et l'allographie. Les opérations de croisement et de transponibilité/traduction des textualisations $n+1$ permettent d'étudier la pratique comme quelque chose qui est encore 
en acte lors du processus d'analyse car l'analyse croise les textualisations, identifie les commensurabilités locales entre les unes et les autres, ainsi qu'elle met en scène les écarts entre les différentes formes de représentation/médiation.

Résumons donc les étapes du parcours que nous avons proposées tout au long de cet article. La démultiplication et diversification des représentations et visualisations permet à la pratique d'être apprivoisée dans ce qu'elle a d'éphémère (1) ; ensuite, elle permet d'être cartographiée par un croisement de points de vue et de spécificités médiumniques (2) ; pour finir, elle est redynamisée à travers la comparaison/ traduction des différentes textualisations et la projection de l'une sur l'autre (3). C'est l'action de projection d'une visualisation sur l'autre qui en permet la confrontation et la traduction à travers une modalité d'analyse qui a le mérite de mimer la pratique-objet d'analyse en reparcourant le croisement non pas des actions, regards, paroles, mais de leur textualisations. Ne pouvant pas rendre compte de la pratique en acte directement, l'analyste doit le faire à travers la mise en relation de ses textualisations et notations qui transforment la pratique en une diagrammatique de médiations qui est par définition une transposition et une recherche de commensurabilité entre densités syntaxiques et de points de vue différents.

Notre proposition méthodologique est enfin une proposition de «fidélité » : il s'agit de concevoir l'analyse comme un processus de traduction, transposition et projection qui puisse rendre compte de la précarité et du caractère provisoire de chaque geste à l'intérieur de la pratique étudiée : ce n'est qu'à travers cette mobilité du point de vue durant l'analyse que l'on pourra en assumer le contrôle, en justifier la syntagmatique et en déceler le sens. 


\section{RÉFÉRENCES}

ANGENOT, V. et al. Sémiotique de la communication en coprésence et à distance: du textualisme à la sémiotique des pratiques. Interfaces numériques, Paris, v. 2, n. 3, p. 531-567, 2013.

BORDRON, J.-F. L'iconicité et ses images. Paris : PUF, 2011.

DONDERO, M. G. Rhétorique et énonciation visuelle. Visible: Rhétorique et visualisation scientifique, Limoges, n. 10, p. 9-31, 2013.

DONDERO, M. G.; FONTANILLE, J. Des images à problèmes: Le sens du visuel à l'épreuve de l'image scientifique. Limoges: Pulim, 2012.

ECO, U. Sémiotique et philosophie du langage. Paris: PUF, 2013 (1984).

. Trattato di semiotica generale. Milan: Bompiani, 1975.

ELSEN, C.; LECLERCQ, P. SketSha ou le pouvoir de l'esquisse comme soutien de la conception collaborative. Lecture Notes in Computer Science, Berlin, n. 5220, p. 20-27, 2008.

FLOCH, J.-M. Êtes-vous arpenteurs ou somnambules? L'élaboration d'une typologie comportementale des voyageurs du métro. In: . Sémiotique, Marketing et Communication. Paris: PUF, 1990. p. 19-48.

FONTANILLE, J. Pratiques sémiotiques. Paris: PUF, 2008.

. Les Espaces subjectifs: Introduction à une sémiotique de l'observateur. Paris: Hachette, 1989.

FONTANILLE, J.; ZILBERBERG, C. Tension et signification. Liège: Mardaga, 1998. 
FOSSALI, P. B. (Org.). Semiotiche: Testo, pratiche, immanenza, 5/06. Torino: Anankè, 2006.

FOSSALI, P. B.; DONDERO, M. G. Sémiotique de la photographie. Limoges: Pulim, 2011.

GOODMAN, N. Languages of Art. London: Bobbs Merrill, 1968.

GOODWIN, C. Practices of Seeing: Visual Analysis. An Ethnomethodological Approach. In.: LEEUWEN, T. van; CAREY, J. (Org.). Il senso del vedere. Roma: Meltemi, 2003.

. Handbook of Visual Analysis. Londres: Sage Publications, 2000. p. 157-182.

. The Blackness of Black: Colour Categories as Situated Practice. In: RESNICK, L. B. et al. (Org.). Discourse, Tools and Reasoning: Essays on Situated Cognition. Berlin - Heidelberg - New York: Springer, 1997.

. Professional Vision. American Anthropologist, Berkeley, v. 96, n. 3, pp. 606-633, 1994.

GREIMAS, A. J. ; COURTÉS, J. Sémiotique: Dictionnaire raisonné de la théorie du langage. Paris: Hachette, 1979.

HUTCHINS, E. Cognition in the wild. Cambridge: MIT Press, 1995.

KAUFMAN, J.-C. L'entretien compréhensif. Paris: Nathan, 1996.

MANETTI, G. La teoria dell'enunciazione: Le origini del concetto e alcuni più recenti sviluppi. Sienne: Protagon, 1998.

MARRONE, G. L'invenzione del testo: Una nuova critica della cultura. Roma-Bari: Laterza, 2010.

METZ, C. L'énonciation impersonnelle ou le site du film. Paris: Klincksieck, 1991. 
MONDADA, L. Chercheurs en interaction: Comment émergent les savoirs. Lausanne: Presses Polytechniques et Universitaires Romandes, 2005.

PAOLUCCI, C. Strutturalismo e interpretazione. Milan: Bompiani, 2010.

RAJEB, S. B.; LECLERCQ P. Collaborations distantes synchrones dans les pratiques de conception. International Journal of Design Sciences and Technology, Paris, p. 29-43, jan. 2013. Disponível em: http://orbi.ulg.ac.be/handle/2268/125521. Acesso em: 23 jun. 2014.

SUCHMAN, L. Plans and situated actions: the problem of human/machine communication. Cambridge: Cambridge University Press, 1987.

VIOLI, P. Il soggetto è negli avverbi: lo spazio della soggettività nella teoria semiotica di Umberto Eco. E/C Rivista dell'AISS Associazione Italiana di Studi Semiotici, Palermo, 07 jan. 2005. Disponível em: http://www.ec-aiss.it/index_d. php?recordID=370. Acesso em: 23 jun. 2014.

Artigo recebido em março de 2014 e aprovado em junho de 2014.

Disponível em: http://seer.fclar.unesp.br/casa 